Check for updates

Cite this: RSC Adv., 2017, 7, 55504

Received 27th October 2017 Accepted 21st November 2017

DOI: 10.1039/c7ra11843k

rsc.li/rsc-advances

\section{High pressure effects on hydrate Cu-BTC investigated by vibrational spectroscopy and synchrotron X-ray diffraction}

\author{
Zhaohui Dong, ${ }^{a}$ Zhongying Mi, (D) *b Weiguang Shi, (D) *c Hui Jiang, ${ }^{a}$ Yi Zheng ${ }^{a}$ \\ and Ke Yang ${ }^{a}$
}

\begin{abstract}
The high pressure behaviors of hydrate Cu-BTC metal-organic framework (MOF) in terms of phase stability, compressibility and reversibility were investigated in situ by synchrotron X-ray powder diffraction as well as vibrational spectroscopy. Two phase transitions, caused by the interaction of water and the sample framework, were revealed by the vibrational spectroscopies. Compressibility of the hydrate Cu-BTC also displays soft and hard regimes, which is the same scenario as non-hydrate Cu-BTC with a pressure transmitting medium. It is further confirmed that the residual water molecules in hydrate Cu-BTC can serve as a pressure transmitting medium with small molecule size under high pressure. Our results not only prove the high stability of Cu-BTC but also provide spectroscopic evidence for the interactions taking place between the guest molecules and the sample framework. Such findings could provide further guidelines for improving Cu-BTC's absorption and storage abilities.
\end{abstract}

\section{Introduction}

Metal organic framework materials (MOFs) are porous materials based on the classical metal ions (such as $\mathrm{Zn}^{2+}, \mathrm{Cu}^{2+}$, and $\mathrm{Al}^{3+}$ ) with electron donors (such as carboxylic acid ester or amine). ${ }^{\mathbf{1}, 2}$ In contrast to aluminosilicate zeolite, MOFs have more tunable architectures and unique chemical properties due to the variable and flexible organic chains in their structures. MOFs, as an intriguing class of porous functional materials, have been extensively studied for decades owing to their promising applications and excellent performance in gas storage, $^{3-8}$ hydrocarbon separation,,$^{9,10}$ catalysis $^{11-15}$ and drug delivery. ${ }^{16-19}$

Normally, the porosity of MOFs, i.e., pore size and geometry, is controlled by synthetic routes, for example, by adjusting the size of the organic linkers. ${ }^{16-19}$ Such modifications are associated with changes in the functional groups. In addition to traditional synthetic routes, high pressure can provide a clean outer force to further tune pore structures without changing the functional groups. The pore structures are very sensitive to pressure, and many meaningful changes have occurred in a relatively low pressure range $(<10 \mathrm{GPa})$; some of them even occur below 2 GPa., ${ }^{\mathbf{1 , 2 0 - 2 6}}$ Previous studies have revealed that

${ }^{a}$ Shanghai Synchrotron Radiation Facility, Shanghai Institute of Applied Physics, CAS, Shanghai, 201204, P. R. China

${ }^{b}$ School of Earth and Space Sciences, Peking University, Beijing, 100871, P. R. China. E-mail: mornsunlight313@gmail.com; Tel: +86-021-33933188

${ }^{c}$ College of Chemistry \& Chemical Engineering, Northeast Petroleum University, Daqing, 163318, P. R. China pressure could induce diverse changes in the pore geometry of MOFs, ${ }^{24,27}$ which consequently affect the absorption capacity, absorption selectivity, and access to binding sites. In addition to the pore geometry, how the pore traps or interacts with guest molecules under high pressure also affects MOFs' absorption abilities. ${ }^{23}$ Thus, exploring the pressure dependence of MOFs' functionality is crucial to develop application-specific products.

One of the common phenomena observed in MOFs is pressure induced amorphization (PIA). For example, in ZIF-8 (ref. 24) and MOF-5, ${ }^{27}$ PIA was observed at $0.34 \mathrm{GPa}$ and $0.35 \mathrm{GPa}$, respectively. A similar scenario was also found in single crystal ZIF-4 but at a higher pressure, between $5.1 \mathrm{GPa}$ and $6.5 \mathrm{GPa}{ }^{1}$ In addition to PIA, pressure induced lattice rearrangement was also found in MOFs, normally below $1 \mathrm{GPa}$. For instance, ZIF-4 underwent a pressure induced phase transformation from orthorhombic to monoclinic phase between $0.13 \mathrm{GPa}$ and $0.56 \mathrm{GPa}^{1}{ }^{1}$ Spencer et al. observed a crystalline to crystalline phase transition occurring bin the range of 0.5-0.8 GPa using neutral zinc imidazolate $\left(\mathrm{Zn}(\mathrm{Im})_{2}\right){ }^{26}$ It is widely recognized that the stability and mechanical properties of MOFs make them an advanced research hot spot owing to their rigid and soft regions in a group of materials which flex and squeeze upon compression. Previous high pressure studies have revealed that MOFs have high compressibility comparable to molecular crystals. Bulk moduli of most MOFs fall in the range of 5-30 GPa, such as

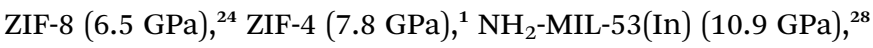
ZAG-4 $(11.7 \mathrm{GPa}),{ }^{29},\left[\mathrm{tmenH}_{2}\right]\left[\mathrm{Er}(\mathrm{HCOO})_{4}\right]_{2}(13.8 \mathrm{GPa}),{ }^{30} \mathrm{Zn}(\mathrm{Im})_{2}$ $(14 \mathrm{GPa}),{ }^{21} \mathrm{LiB}(\mathrm{IM})_{4}(16.6 \mathrm{GPa}),{ }^{21}$ and MOF-5 (16.5 GPa). ${ }^{27}$

$\mathrm{Cu}$ based MOF is a commercially available highly porous material named copper benzene-1,3,5-tricarboxylate (Cu-BTC). 
It has been proposed as a suitable compound for $\mathrm{CO}_{2}$ capture and gas separation purposes. ${ }^{\mathbf{1 0 , 3 1}}$ However, few high pressure experiments have been carried out on Cu-BTC. Moreover, few studies of $\mathrm{Cu}$-BTC showed excellent results in comparison with other MOFs mentioned above. Chapman et al. documented a change in the equation of state in Cu-BTC upon compression by introducing different pressure transmitting media into the sample. ${ }^{23,25}$ Moreover, Cu-BTC exhibited an intrinsic property of high resistance to pressure when it was continually compressed up to $8 \mathrm{GPa} .{ }^{25}$ However, no high pressure study was carried out beyond $8 \mathrm{GPa}$, leaving the stability and high pressure behaviors of Cu-BTC beyond 8 GPa still unclear.

In this study, we extended the high pressure region of $\mathrm{Cu}$ BTC up to $20 \mathrm{GPa}$. High pressure behaviors of Cu-BTC were investigated by in situ Raman spectroscopy, IR spectroscopy and $\mathrm{X}$-ray diffraction with synchrotron radiation. Structural stability, compressibility, and phase transition reversibility were examined. By employing vibrational spectroscopy in addition to XRD, we expect to gain a better understanding of the extraordinary properties of $\mathrm{Cu}$-BTC porous materials under high pressure, particularly the interactions taking place between guest molecules and sample frameworks.

\section{Experiments}

Copper benzene-1,3,5-tricarboxylate (Cu-BTC) was purchased from Sigma Aldrich. The powder Cu-BTC sample was used without further treatment. Morphologies of $\mathrm{Cu}$-BTC before and after compression were examined by scanning electronic microscopy (SEM, FEI 1540XB FIB/SEM). In situ structural characterization was performed using vibrational spectroscopy and synchrotron X-ray diffraction (XRD).

In situ high pressure Raman and XRD measurements were carried out using a symmetric diamond anvil cell (DAC) with a pair of type I diamonds. The diamond anvils have culet size of $500 \mu \mathrm{m}$. A stainless steel gasket with a hole of $180 \mu \mathrm{m}$ drilled in the center was used as the sample chamber. For the in situ infrared studies, a DAC equipped with a pair of $400 \mu \mathrm{m}$ type II diamonds was used. Similar to the Raman and XRD measurements, a $180 \mu \mathrm{m}$ hole drilled in a stainless steel gasket was used as the sample chamber. For all experiments, the pressure was determined by the well-established ruby fluorescence method, ${ }^{32}$ and no pressure transmitting medium was used.

The Raman spectra were collected using a Renishaw inVia Raman microscopy system with a $633 \mathrm{~nm}$ line excited by a HeNe laser. The infrared measurements were carried out on a Bruker VERTEX 70v FTIR spectrometer and a custom IR microscope at the Center for High Pressure Science and Technology Advanced Research, Beijing.

The angle dispersive XRD synchrotron experiments were accomplished at the 12.2.2 beamline at the Advanced Light Source (ALS) at Lawrence Berkeley Laboratory (LBL), as well as the BL15U beamline at Shanghai Synchrotron Facility (SSRF). High pressure XRD experiments at ALS were conducted using a monochromatic beam with a wavelength of $0.4132 \mathrm{~nm}(30$ $\mathrm{keV}$ ) and a focused beam size of $10 \times 10 \mu \mathrm{m}^{2}$. A MAR CCD detector was used to collect the 2D Debye-Scherrer patterns.
The diffraction parameters were calibrated using $\mathrm{LaB}_{6}$ standard pattern. XRD measurements at SSRF were conducted using a $0.6188 \mathrm{~nm}$ monochromatic beam. $\mathrm{CeO}_{2}$ was used as the calibrant for experimental parameters. The beam size was focused down to $5 \times 4 \mu^{2}$ by a K-B mirror. The $2 \mathrm{D}$ Debye-Scherrer diffraction patterns were integrated using Fit2D program for further analysis.

\section{Results and discussions}

\subsection{Characterization of Cu-BTC at ambient pressure}

The porous framework of Cu-BTC with cubic structure $(F m \overline{3} m)$ is depicted in Fig. 1. $\mathrm{Cu}$ (II) ions form bridges with the carboxylate groups on discrete 1,3,5-benzenetricarboxylate (BTC) ligands. Two adjacent $\mathrm{Cu}(\mathrm{II})$ ions constitute $\mathrm{Cu}$ (II) dimers. Such $\mathrm{Cu}$ (II) dimers link via the trigonal BTC molecules, and form extended three dimensional (3D) networks. The 3D network consists of three types of cages with different sizes, among which the largest has a diameter of $10 \AA^{33} .^{33}$ The SEM result shows a particle size of tens of microns (Fig. 2).

The vibrational spectra of $\mathrm{Cu}-\mathrm{BTC}$ at ambient conditions are shown in Fig. 3. In the Raman spectrum (Fig. 3a), regions of 1200-1400 $\mathrm{cm}^{-1}$ and $>1800 \mathrm{~cm}^{-1}$ are excluded due to the intense Raman peaks of diamond at $1335 \mathrm{~cm}^{-1}$ and $2400 \mathrm{~cm}^{-1}$. Raman peaks located at $1618 \mathrm{~cm}^{-1}$ and $1006 \mathrm{~cm}^{-1}$ are associated with the benzene ring stretch modes, labeled as $\nu_{\mathrm{C}=\mathrm{C}(\mathrm{Ar})}$ in the figure. The peaks at $743 \mathrm{~cm}^{-1}$ and $830 \mathrm{~cm}^{-1}$ are assigned to out-of-plane ring bending $\left(\delta_{\mathrm{C}=\mathrm{C}(\mathrm{Ar})}\right)$ and out-of-plane $\mathrm{C}-\mathrm{H}$ bending of the benzene ring $\left(\delta_{\mathrm{C}-\mathrm{H}(\mathrm{Ar})}\right)$. The double peaks at $1465 \mathrm{~cm}^{-1}$ and $1548 \mathrm{~cm}^{-1}$ are ascribed to the symmetric and asymmetric stretching modes of the carboxylate groups, which are labeled as $\nu_{\mathrm{sym} \mathrm{COO}^{-}}$and $\nu_{\text {asym } \mathrm{COO}^{-}}$, respectively. In the low frequency region (100-600 $\left.\mathrm{cm}^{-1}\right)$, two peaks are observed at $191 \mathrm{~cm}^{-1}$ and $501 \mathrm{~cm}^{-1}$, which are associated with the $\mathrm{Cu}-\mathrm{O}$ stretch. As shown in Fig. 1, there are two similar $\mathrm{Cu}-\mathrm{O}$ bonds in the frame network. The $\mathrm{Cu}-\mathrm{O} 1$ lies on the equatorial position, while the $\mathrm{Cu}-\mathrm{O} 2$ bond lies on the axial position. The bond lengths for $\mathrm{Cu}-\mathrm{O} 1$ and $\mathrm{Cu}-\mathrm{O} 2$ are obtained as $1.941 \AA$ and 2.277 $\AA$, respectively. Referring to the Hooke's law, the $\mathrm{Cu}-\mathrm{O} 1$ stretch should be obtained at a higher frequency. Therefore, the two Raman modes observed at $501 \mathrm{~cm}^{-1}$ and $191 \mathrm{~cm}^{-1}$ are relevant to the equatorial and axial $\mathrm{Cu}-\mathrm{O}$, respectively, which is consistent with a previous study. ${ }^{34}$

The IR spectrum of the sample is shown in Fig. $3 \mathrm{~b}$. The spectrum can be divided into three regions. The first is the region below $1150 \mathrm{~cm}^{-1}$, in which most vibrational modes of the BTC ligand are detected. The two bands at $730 \mathrm{~cm}^{-1}$ and $758 \mathrm{~cm}^{-1}$ are the out-of-plane $\mathrm{C}-\mathrm{H}$ bending modes, while the band at $1113 \mathrm{~cm}^{-1}$ represents the in-plane $\mathrm{C}-\mathrm{H}$ bending mode, all of which are associated with the aromatic ring of the BTC. ${ }^{35,36}$ The second region is from $1150 \mathrm{~cm}^{-1}$ to $1700 \mathrm{~cm}^{-1}$, in which the characteristic bands associated with the carboxylate groups of the BTC ligand are detected. The weak peaks at $1191 \mathrm{~cm}^{-1}$ and $1274 \mathrm{~cm}^{-1}$ are attributed to the $\mathrm{C}-\mathrm{O}$ stretching modes. The intense peaks at $1373 / 1449 \mathrm{~cm}^{-1}$ are assigned to the asymmetric stretching of the carboxylate groups, while the peaks at $1571 / 1630 \mathrm{~cm}^{-1}$ belong to their symmetric stretching. More 


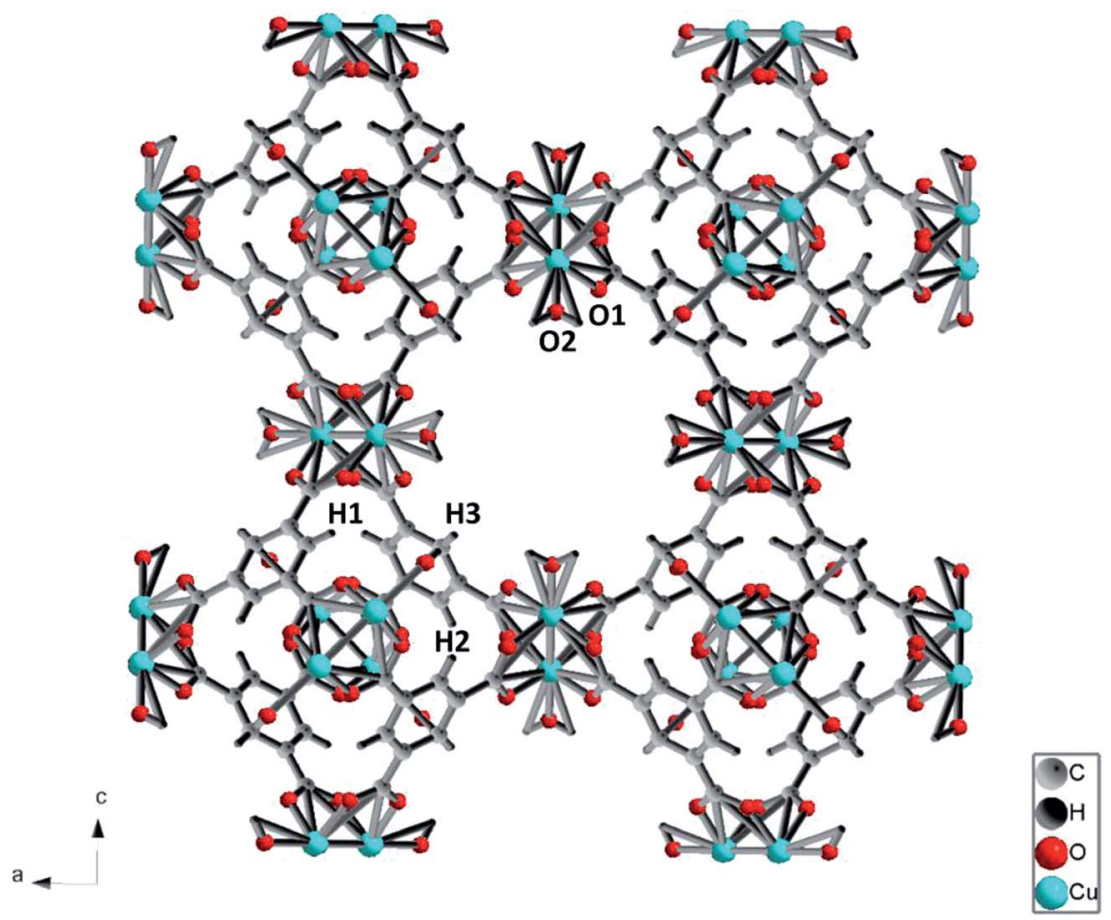

Fig. 1 A presentation of the structure of $\mathrm{Cu}-\mathrm{MOF}$ frame work. Color code: $\mathrm{Cu}$ atoms, green; $\mathrm{O}$ atoms, red; $\mathrm{H}$ atoms, dark grey; $\mathrm{C}$ atoms, grey. O1 and $\mathrm{O} 2$ denote oxygen atoms attached to $\mathrm{Cu}$ atom at equatorial and axial position, respectively.

precisely, the two peaks at $1449 \mathrm{~cm}^{-1}$ and $1571 \mathrm{~cm}^{-1}$ are attributed to the $\mathrm{C}-\mathrm{C}$ stretching associated with the aromatic rings. The third region is above $2800 \mathrm{~cm}^{-1}$, in which the broad $\mathrm{O}-\mathrm{H}$ stretch mode of $\mathrm{H}_{2} \mathrm{O}$ is observed. In addition, the $\mathrm{O}-\mathrm{H}$ bending mode is detected at $1241 \mathrm{~cm}^{-1} \cdot{ }^{37}$ The existence of $\mathrm{O}-\mathrm{H}$ bending and stretching modes confirms that the starting sample contained a large amount of water. The IR modes associated with $\mathrm{Cu}$ lie below $600 \mathrm{~cm}^{-1}$, 36 which are out of the detectable range of the IR system.

\subsection{Raman results of Cu-BTC upon compression and decompression}

A Cu-BTC sample was studied under high pressure upon compression and decompression by Raman spectroscopy. As shown in Fig. 4, the Raman profile remains unchanged up to $9 \mathrm{GPa}$, and then a shoulder peak is found at $830 \mathrm{~cm}^{-1}$. Peaks below $600 \mathrm{~cm}^{-1}$, associated with $\mathrm{Cu}-\mathrm{O}$ vibration, completely vanish and a new broad peak at $280 \mathrm{~cm}^{-1}$ appears. This new peak could be assigned to the absent $\mathrm{Cu}-\mathrm{Cu}$ stretching of $\mathrm{Cu}(\mathrm{II})$ dimers in the framework, which could be enhanced and become visible due to the cell shrinkage at high pressure. Moreover, two new peaks are observed at $675 \mathrm{~cm}^{-1}$ and $804 \mathrm{~cm}^{-1}$, and they become distinguishable beyond $9 \mathrm{GPa}$. Based on assignment, the Raman region of $600 \mathrm{~cm}^{-1}$ to $1200 \mathrm{~cm}^{-1}$ belongs to vibrations associated with benzene rings such as ring bending and ring related $\mathrm{C}-\mathrm{H}$ stretching. ${ }^{3}$ The changes occuring in this region under high pressure strongly suggest that the benzene ring could interact with its surrounding atoms or guest molecules. In conclusion, the apparent changes in the Raman profile imply a possible phase transition occurring at $9 \mathrm{GPa}$. Upon compression, no more changes are observed up to $15 \mathrm{GPa}$.

Examination of the pressure effects on the crystal reversibility of $\mathrm{Cu}$-BTC were also conducted upon decompression to


Fig. 2 SEM images of Cu-BTC taken before (a) and after (b) compression. 

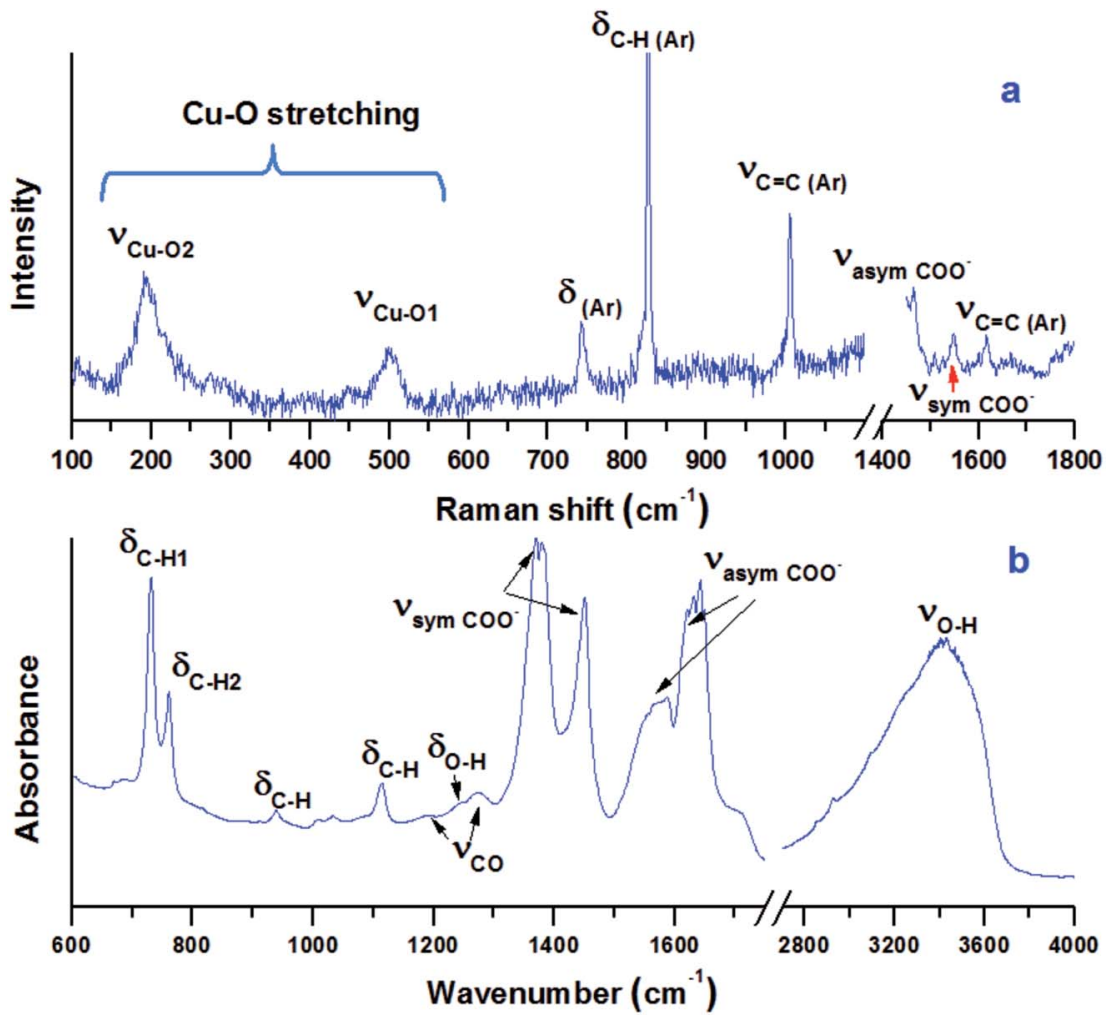

Fig. 3 Raman (a) and IR (b) spectra of Cu-BTC obtained at ambient condition in a DAC. Both Raman and IR peaks are assigned and labeled. $\delta$ stands for bending, while $\nu$ denotes stretching. Ar stands for the benzene ring.

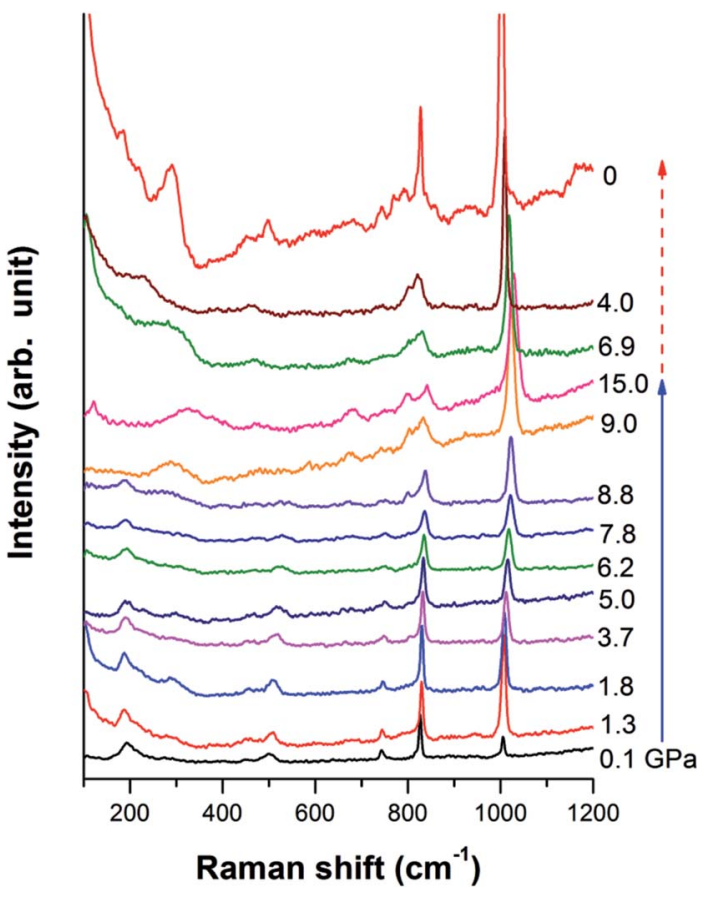

Fig. 4 Selected Raman spectra of Cu-BTC upon compression and decompression. Pressure collected for each spectrum is labeled. The solid and dashed lines indicate the compression and decompression sequence, respectively. All the spectra are offset for clarity. gain important information on the transformation mechanism. In general, the intensity of all the Raman peaks increases gradually as pressure decreases, and all the Raman modes shift to lower frequencies. The three new peaks, which appear at high pressure, gradually disappear as pressure releases. When the pressure is completely removed, all Raman peaks observed before compression are recovered, indicating that the phase transition is reversible.

\subsection{FTIR results of $\mathrm{Cu}-\mathrm{BTC}$ upon compression and decompression}

In addition to Raman measurements, the sample was also studied by in situ FTIR to investigate asymmetric vibrations. Fig. 5 a presents behaviours of out-of-plane $\mathrm{C}-\mathrm{H}$ bending modes $\delta_{\mathrm{C}-\mathrm{H}}$ upon compression. The two peaks remain steady up to $16 \mathrm{GPa}$ and then merge upon further compression. As shown in Fig. 5b, the $\delta_{\mathrm{CH}}$ band at $938 \mathrm{~cm}^{-1}$ split into two observed peaks at $930 \mathrm{~cm}^{-1}$ and $958 \mathrm{~cm}^{-1}$ starting at $4.1 \mathrm{GPa}$, which could be attributed to the interaction between water and the framework. At the same pressure, the $\nu_{\mathrm{CO}}$ modes as well as the $\mathrm{O}-\mathrm{H}$ bending mode are completely suppressed. The new peak at $930 \mathrm{~cm}^{-1}$ remains steady up to $19.2 \mathrm{GPa}$, the highest pressure achieved, while the peak at $958 \mathrm{~cm}^{-1}$ disappears at $17.4 \mathrm{GPa}$ due to pressure suppression. Moreover, the weak $\nu_{\mathrm{C}-\mathrm{H}(\mathrm{Ar})}$ mode of the aromatic ring disappears at $4.1 \mathrm{GPa}$. In addition to the peak splitting of $\delta_{\mathrm{CH}}$ and suppression of $\nu_{\mathrm{CO}}$, these observations suggest a possible on-site phase transition. The $\delta_{\mathrm{CH}}$ mode found 



Fig. 5 IR spectra of Cu-BTC collected in different regions upon compression, where (a), (b), (c) denote ranges below $800 \mathrm{~cm}^{-1}, 800-1350 \mathrm{~cm}^{-1}$, and $1300-3800 \mathrm{~cm}^{-1}$, respectively. Pressure for each spectrum is labelled. The dot dashed line in (c) is drawn for eye guidance. The IR spectra at the bottom of each figure are of the retrieved sample after compression. All the spectra are offset for clarity.

at $1113 \mathrm{~cm}^{-1}$ can still be clearly discerned at $19.2 \mathrm{GPa}$. The symmetric and asymmetric stretching modes of carboxylate groups (in Fig. 5c) are very stable up to $19.2 \mathrm{GPa}$ with slightly decreased intensity and broadened profile. Upon compression, the $\nu_{\mathrm{O}-\mathrm{H}}$ mode exhibits a red shift, indicating bond softening of the hydrogen bond. It is suggested that $\mathrm{H}_{2} \mathrm{O}$ interacts with its environment (e.g., the Cu-MOF framework) upon compression owing to weakening of hydrogen bonds. At $19.2 \mathrm{GPa}$, the highest pressure achieved in this study, the characteristic IR peaks are still clearly discernable, suggesting that no complete PIA occurred in $\mathrm{Cu}-\mathrm{BTC}$, which is further confirmed by the subsequent XRD results.

Upon decompression, the IR spectrum of the retrieved sample shows an almost identical profile as the one recorded before compression, indicating that the phase transition is completely reversible, which is consistent with the Raman results.

\subsection{XRD results of $\mathrm{Cu}-\mathrm{BTC}$ upon compression and decompression}

Fig. 6 shows the structural evolution of Cu-BTC under high pressure. The XRD results confirm that $\mathrm{Cu}-\mathrm{BTC}$ originally has a cubic structure with cell parameters of $a=26.45 \AA$ at ambient pressure, which is consistent with reference value. ${ }^{34}$ Upon compression, no significant change is observed in the XRD pattern, except the suppression in intensity of all reflections. Such observation suggests that no structural change occurs in $\mathrm{Cu}-\mathrm{BTC}$ upon compression. As the pressure is increased to 14.6 GPa, reflections below $6^{\circ}$ are still clearly observed, indicating the sample is still in a crystalline phase.

Upon decompression, reflections of high $2 \theta\left(>6^{\circ}\right)$ that vanished at high pressure reappear. The XRD pattern of the recovered sample has the same profile as the starting material, which confirms that the recovered sample regains its original structure. However, in comparison with the XRD pattern taken at $1.1 \mathrm{GPa}$, the intensity of each reflection is weaker and slightly broadened. In addition, the diffraction background is also

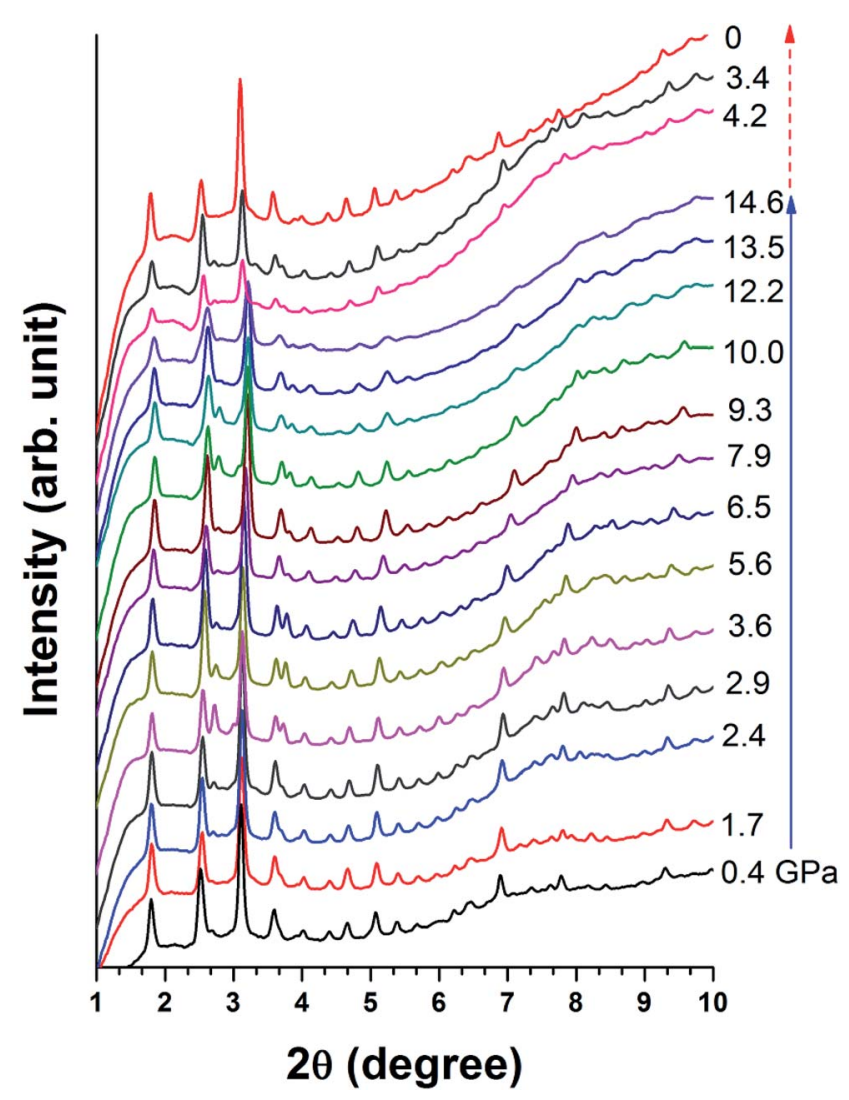

Fig. 6 Collected XRD patterns for Cu-BTC upon compression and decompression. The experimental pressure is labeled beside each pattern in GPa. The solid and dashed arrows indicate the compression and decompression sequences, respectively. 
higher. Morphology of the recovered sample was also examined by SEM as shown in Fig. 2b, in which the particle size of Cu-BTC is significantly reduced. Such reduction in particle size and pressure induced sample disorder are attributed to the diffraction profile broadening.

\subsection{Discussion}

The pressure dependences for all Raman modes are shown in Fig. 7. With a linear fitting, the shifting rate $(\Delta \nu / \Delta p)$ for each Raman mode was obtained and labeled. Upon compression, almost all Raman modes associated with organic linkers shift to higher frequencies at a similar rate of $\sim 1 \mathrm{~cm}^{-1} \mathrm{GPa}^{-1}$ except the two $\mathrm{Cu}-\mathrm{O}$ stretching modes $\left(\nu_{\mathrm{Cu}-\mathrm{O} 1}\right.$ and $\left.\nu_{\mathrm{Cu}-\mathrm{O} 2}\right)$. The $\nu_{\mathrm{Cu}-\mathrm{O} 1}$ mode shifts at a rate of $3.7 \mathrm{~cm}^{-1} \mathrm{GPa}^{-1}$, which is two times more sensitive than the rest. In strong contrast, the $\nu_{\mathrm{Cu}-\mathrm{O} 2}$ mode is insensitive to pressure, and even has a negative pressure dependence. Such striking difference is ascribed to the different locations of the two bonds in the framework of Cu-BTC. As shown in Fig. 1, the $\mathrm{Cu}-\mathrm{O} 1$ and $\mathrm{Cu}-\mathrm{O} 2$ bonds lie in the equatorial and axial positions, respectively. In contrast to $\mathrm{Cu}-\mathrm{O} 1$, the axial position gives the $\mathrm{Cu}-\mathrm{O} 2$ bond more room to respond to pressure. ${ }^{5}$ Accordingly, the $\mathrm{Cu}-\mathrm{O} 2$ is least sensitive to compression. Moreover, compared to their original values, bond shortening and bond lengthening are respectively observed for $\mathrm{Cu}-\mathrm{O} 1(1.700 \AA$ vs. $1.941 \AA)$ and $\mathrm{Cu}-\mathrm{O} 2(2.410 \AA$ vs. $2.277 \AA$ ) upon compression, which directly attribute to the red and blue shift of the $\mathrm{Cu}-\mathrm{O} 1$ and $\mathrm{Cu}-\mathrm{O} 2$, respectively, under high pressure. It is well known that chemical bonds shrink under high pressure. However, it is observed that the $\mathrm{Cu}-\mathrm{O} 1$ bond softens under pressure, probably owing to the interactions between the $\mathrm{Cu}-\mathrm{O}$ bond and surrounding atoms. In this study, the starting Cu-BTC is found to contain a large amount of residual water. Furthermore, the changes observed in Raman results are primarily related to the $\mathrm{Cu}-\mathrm{O} 1$ bonds. It is reasonable to speculate that during compression the residual water was squeezed into cages and interacted with the $\mathrm{Cu}-\mathrm{O}$ bond, which will be discussed in subsequent sections.

Similar to Raman modes, IR modes of Cu-BTC also gradually shift to higher wavenumbers upon compression except the doublet peak $\delta_{\mathrm{C}-\mathrm{H} 1}$ and $\delta_{\mathrm{C}-\mathrm{H} 2}$. For comparison purposes, pressure dependences of $\delta_{\mathrm{C}-\mathrm{H} 1}$ and $\delta_{\mathrm{C}-\mathrm{H} 2}$ modes are plotted in Fig. 7. It is clearly shown that the $\delta_{\mathrm{C}-\mathrm{H} 1}$ is more sensitive to pressure than $\delta_{\mathrm{C}-\mathrm{H} 2}$ mode $\left(1.05 \mathrm{~cm}^{-1} \mathrm{GPa}^{-1}\right.$ vs. $\left.-0.09 \mathrm{~cm}^{-1} \mathrm{GPa}^{-1}\right)$. As shown in Fig. 1, benzene rings with carbonyl groups form the cage structures. Hydrogen atoms located in three different positions point to different cages, where $\mathrm{H} 1$ and $\mathrm{H} 2$ have the same chemical atmosphere. Thus, $\delta_{\mathrm{C}-\mathrm{H} 1}$ and $\delta_{\mathrm{C}-\mathrm{H} 2}$ are assigned to the bending of $\mathrm{C}-\mathrm{H} 1 / \mathrm{H} 2$ and $\mathrm{C}-\mathrm{H} 3$ bonds respectively. Moreover, the area integral ratio of $A \delta_{\mathrm{C}-\mathrm{H} 1} / A \delta_{\mathrm{C}-\mathrm{H} 2}$ is 2.11 , which further confirms the amount of $\mathrm{C}-\mathrm{H} 1$ is twice that of $\mathrm{C}-\mathrm{H} 2$. Therefore, we can ascribe the different pressure sensitivities of $\delta_{\mathrm{C}-\mathrm{H}}$ to their different environments, where the $\mathrm{C}-\mathrm{H} 3$ has more space to collide or interact with its surroundings upon compression.

In this study, we observed that the phase transition results are inconsistent among the different probe techniques. In the results obtained from vibrational spectroscopy, one phase transition is observed for both Raman and IR measurements,


Fig. 7 Pressure dependences of Raman (left) and IR (right) modes in Cu-BTC. Different symbols denote different Raman/IR modes which are labeled. Shift rate of each mode is also labeled. 
but at different pressures. In Raman results, the phase transition is found at $\sim 9 \mathrm{GPa}$, which was deduced from peak altering in the $\mathrm{Cu}-\mathrm{O}$ and aromatic ring vibrational regions. In contrast, the phase transition in IR results occurred between 4.1 and 5.7 GPa, which was suggested by a series of IR mode changes associated with the framework. However, in strong contrast to both Raman and IR, no phase transition was found from the XRD patterns. The absence of the two phase transitions in XRD results strongly suggests that the phase transitions observed in Raman and IR only involve chemical environment changes without any crystal structural alteration. Thus, no crystal phase transition is observed in Cu-BTC using either vibrational spectroscopy or XRD. The inconsistency between Raman and IR is probably due to the differences between Raman and IR techniques. It is well known that IR has a higher sensitivity to asymmetric vibrational changes due to the weaker scattering effect of Raman technique. Therefore, the lower phase transition observed in IR could not be revealed by Raman because of the weak interactions.

However, as shown in Fig. 8, the pressure dependence of the cell parameters clearly reveals two transitions at $\sim 5.8 \mathrm{GPa}\left(P_{\mathrm{t} 1}\right)$ and $10 \mathrm{GPa}\left(P_{\mathrm{t} 2}\right)$. Such transitions are known as compressibility changes that are not accompanied by any changes in the crystal structure. The $P_{\mathrm{t} 1}$ compressibility transition is the same as that reported in a previous high-pressure study of Cu-BTC. ${ }^{25}$ Chapman et al. have studied non-hydrate Cu-BTC with different pressure transmitting media, and concluded that this compressibility transition only occurred in Cu-BTC with small molecule alcohol-based pressure transmitting media (e.g. water, MEW and isopropanol). ${ }^{25}$ No apparent transition was observed in Cu-BTC without PTM or in large molecule Fluorinert fluid. In addition, it has been proposed that residual water in a hydrate

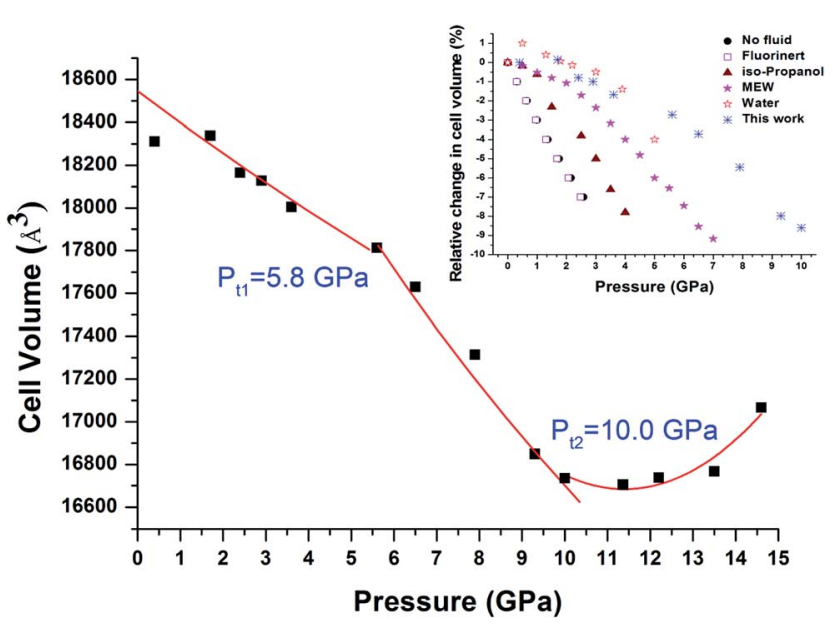

Fig. 8 Pressure dependence of the unit cell volume for Cu-BTC. Square solid symbols are the experimental data obtained upon compression, respectively, from this study. Experimental data points in the regions $0-P_{\mathrm{t} 1}$ and $P_{\mathrm{t} 1}-P_{\mathrm{t} 2}$ are fitted with the third Birch Murnaghan equation of state. The solid line in the region of $>P_{\mathrm{t} 2}$ is only for eye guidance. The inset figure is relative change of cell volume of $\mathrm{Cu}$-BTC with different pressure transmitting media adopted from references. The data of $\mathrm{Cu}-\mathrm{BTC}$ with water are from ref. 23 . The rest are all adopted from ref. 25. sample could act as a pressure transmitting medium..$^{23}$ In this study, the starting Cu-BTC was confirmed to contain a large amount of water. Compared with results obtained by Chapman et al., our results indicate the same scenario as that in $\mathrm{Cu}-\mathrm{BTC}$ with water. Therefore, our findings further confirm that the residual water acted as the guest molecules upon compression. Furthermore, as shown in Fig. 8, the compressibility transition pressure varies as the PTM changes. The Cu-BTC with water demonstrates the highest pressure compressibility transition pressure compared with other hydrostatic pressure media including MEW, isopropyl alcohol and Fluorinert. The compressibility transition pressure increases in the order of no$\mathrm{PTM} \rightarrow$ Fluorinert $\rightarrow$ isopropyl alcohol $\rightarrow$ MEW $\rightarrow$ water as the molecular size decreases, which strongly suggests that the compressibility transition pressure is dependent on the molecular size of the hydrostatic fluid used.

For comparison, bulk moduli for the first two regions, $0-P_{\mathrm{t} 1}$ and $P_{\mathrm{t} 1}-\mathrm{P}_{\mathrm{t} 2}$, were obtained by fitting the experimental data points using the third Birch Murnaghan equation of state with $B^{\prime}{ }_{0}$ fixed at 4 . The obtained bulk moduli are $122.6 \mathrm{GPa}$ and $38.2 \mathrm{GPa}$ for regions $0 \mathrm{GPa}-P_{\mathrm{t} 1}$ and $P_{\mathrm{t} 1}-P_{\mathrm{t} 2}$, respectively, which are considered hard and soft regimes. As shown in Table 1, the values obtained are slightly higher, but still reasonable in comparison with values reported by Chapman et al. considering the sample and experimental difference. ${ }^{25}$ Such results support the theory that the hard regimes are present due to the uptaking of guest molecules, which helps to sustain the framework under high pressure. ${ }^{25}$ The bulk moduli in the soft regime are all similar to the bulk modulus of pure Cu-BTC without pressure transmitting medium, indicating that compressibility in the soft regime can be attributed to intrinsic properties of the framework itself, independent from the type of pressure transmitting medium used. ${ }^{25}$

Moreover, it is interesting that $P_{\mathrm{t} 1}$ and $P_{\mathrm{t} 2}$ are respectively coincident with phase transition pressures observed in IR and Raman results, taking experimental error into consideration. As mentioned earlier, below $P_{\mathrm{t} 1}$, the sample is in the process of uptaking water into its cages. High pressure study of $\mathrm{H}_{2} \mathrm{O}$ insertion in $\mathrm{AlPO}_{4}-54 \cdot x \mathrm{H}_{2} \mathrm{O}$ has revealed that $\mathrm{H}_{2} \mathrm{O}$ molecules tended to concentrate near the pore walls once entering the pores. ${ }^{5}$ In this study, the first phase transition $P_{\mathrm{t} 1}$ observed in IR is deduced by vibrational changes linked to the BTC, which constitutes the main part of the framework wall. Therefore, it is reasonable to speculate that during the $0-P_{\mathrm{t} 1}$ pressure period, water molecules

Table 1 Bulk moduli $\left(B_{0}\right)$ and compressibility transition pressure of Cu-BTC with different transmitting media

Pressure transmitting

media $\quad P_{\text {trans }}(\mathrm{GPa}) \quad B_{0}$ hard $(\mathrm{GPa}) \quad B_{0 \text { soft }}(\mathrm{GPa})$

None $^{a}$

30.7

Fluorinert $^{a}$

Isopropyl alcohol $^{a}$

Methanol-ethanol-water ${ }^{a}$

Water $^{b}$

$\sim 2.5$

$\sim 5.8$

117.6

116.0

122.6

29.5

25.9

33.6

38.1 
could also accumulate in the pore particularly along the framework wall. Below $P_{\mathrm{t} 1}$, the interaction is too weak to be detected by Raman spectroscopy. At $P_{\mathrm{t} 2}$, pressure induced sample shrinking becomes substantial enough that interactions and $\mathrm{Cu}-\mathrm{Cu}$ stretching of the $\mathrm{Cu}$ (II) dimer can be detected by Raman. However, the $\mathrm{Cu}$ associated IR modes lying below $600 \mathrm{~cm}^{-1}$ could not studied by IR spectroscopy due to the equipment edge limitation. Thus, the phase transition occurring at $P_{\mathrm{t} 2}$ is missing in our IR results.

A pressure induced volume expansion is observed at pressure $>P_{\mathrm{t} 2}$, which is reported for the first time. The phenomenon of negative thermal expansion is speculated to be ascribed to the translation, vibration and twisting vibration of aromatic rings and $\mathrm{Cu}(\mathrm{II})$ dimers, which increases distortion of the neighboring two units and further induces negative thermal expansion. Future studies are necessary to verify such speculation.

\section{Conclusion}

In conclusion, hydrate Cu-BTC was studied under high pressure up to $20 \mathrm{GPa}$ without any pressure transmitting medium by vibrational spectroscopy and XRD with synchrotron radiation. The hydrate Cu-BTC shows strong stability up to $20 \mathrm{GPa}$ with no structural change. Vibrational spectroscopy successfully revealed two non-crystal structural phase transitions $(\sim 5.8$ and $9 \mathrm{GPa}$ ) caused by interaction changes between water and sample framework. These two phase transition pressures are consistent with the compressibility transition observed in XRD results, which together confirm that the sample underwent a water uptake process and then a pressure induced compression. Moreover, our finding also proves the compressibility transition pressure is dependent on the molecular size of the pressure transmitting medium, while the compressibility was an intrinsic property of the Cu-BTC framework, independent from the experimental conditions. This work not only helps to study the mechanical stability of Cu-BTC, but also provides spectroscopic evidence to understand the interaction between the framework and guest molecules.

\section{Conflicts of interest}

There are no conflicts to declare.

\section{Acknowledgements}

This work was supported by a National Natural Science Foundation of China (\#11404362), and the Shanghai Sailing Plan (14YF1407400). We acknowledge Dr Dongxu Liang, Dr Shangming He for technique support at BL15U1 at Shanghai Synchrotron Facility. We would also thank Dr Martin Kunz and Dr Jinyuan Yan at beamline 12.2.2 at ALS for their kindly technique support. We appreciate Dr Haiyan Zheng's help with Infrared spectroscopy measurements.

\section{References}

1 T. D. Bennett, P. Simoncic, S. A. Moggach, F. Gozzo, P. Macchi, D. A. Keen, J.-C. Tan and A. K. Cheetham, Chem. Commun., 2011, 47, 7983-7985.

2 K. W. Chapman, P. J. Chupas and C. J. Kepert, J. Am. Chem. Soc., 2005, 127, 15630-15636.

3 P. B. Somayajulu Rallapalli, M. C. Raj, D. V. Patil, K. P. Prasanth, R. S. Somani and H. C. Bajaj, Int. J. Energy Res., 2013, 37, 746-753.

4 E. Deniz, F. Karadas, H. A. Patel, S. Aparicio, C. T. Yavuz and M. Atilhan, Microporous Mesoporous Mater., 2013, 175, 34-42.

5 Y. Peng, V. Krungleviciute, I. Eryazici, J. T. Hupp, O. K. Farha and T. Yildirim, J. Am. Chem. Soc., 2013, 135, 11887-11894.

6 R. Krishna and J. M. van Baten, Sep. Purif. Technol., 2012, 87, 120-126.

7 W. Wong-Ng, I. Levin, J. A. Kaduk, L. Espinal and H. Wu, J. Alloys Compd., 2016, 656, 200-205.

8 Y. Hu, B. Lin, P. He, Y. Y. Li, Y. N. Huang and Y. Song, Chem.Eur. J., 2015, 21, 18739-18748.

9 V. F. D. Martins, A. M. Ribeiro, A. Ferreira, U. H. Lee, Y. K. Hwang, J.-S. Chang, J. M. Loureiro and A. E. Rodrigues, Sep. Purif. Technol., 2015, 149, 445-456.

10 G. Autie-Castro, M. A. Autie, E. Rodriguez-Castellon, C. Aguirre and E. Reguera, Colloids Surf., A, 2015, 481, 351357.

11 M. Alvaro, E. Carbonell, B. Ferrer, F. X. Llabrés i Xamena and H. Garcia, Chem.-Eur. J., 2007, 13, 5106-5112.

12 M. A. Nasalevich, M. G. Goesten, T. J. Savenije, F. Kapteijn and J. Gascon, Chem. Commun., 2013, 49, 10575-10577.

13 K. Schlichte, T. Kratzke and S. Kaskel, Microporous Mesoporous Mater., 2004, 73, 81-88.

14 J. Kim, H.-Y. Cho and W.-S. Ahn, Catal. Surv. Asia, 2012, 16, 106-119.

15 R.-Q. Zou, H. Sakurai and Q. Xu, Angew. Chem., Int. Ed., 2006, 45, 2542-2546.

16 P. Horcajada, C. Serre, G. Maurin, N. A. Ramsahye, F. Balas, M. Vallet-Regi, M. Sebban, F. Taulelle and G. Ferey, J. Am. Chem. Soc., 2008, 130, 6774-6780.

17 J. Gordon, H. Kazemian and S. Rohani, Mater. Sci. Eng., C, 2015, 47, 172-179.

18 V. André, S. Quaresma, J. L. F. da Silva and M. T. Duarte, Beilstein J. Org. Chem., 2017, 13, 2416-2427.

19 P. Horcajada, T. Chalati, C. Serre, B. Gillet, C. Sebrie, T. Baati, J. F. Eubank, D. Heurtaux, P. Clayette, C. Kreuz, J. S. Chang, Y. K. Hwang, V. Marsaud, P. N. Bories, L. Cynober, S. Gil, G. Ferey, P. Couvreur and R. Gref, Nat. Mater., 2010, 9, 172-178.

20 Y. Hu, H. Kazemian, S. Rohani, Y. Huang and Y. Song, Chem. Commun., 2011, 47, 12694-12696.

21 T. D. Bennett, J.-C. Tan, S. A. Moggach, R. Galvelis, C. MellotDraznieks, B. A. Reisner, A. Thirumurugan, D. R. Allan and A. K. Cheetham, Chem.-Eur. J., 2010, 16, 10684-10690.

22 A. Clearfield, Dalton Trans., 2016, 45, 4100-4112.

23 A. J. Graham, J. C. Tan, D. R. Allan and S. A. Moggach, Chem. Commun., 2012, 48, 1535-1537. 
24 K. W. Chapman, G. J. Halder and P. J. Chupas, J. Am. Chem. Soc., 2009, 131, 17546-17547.

25 K. W. Chapman, G. J. Halder and P. J. Chupas, J. Am. Chem. Soc., 2008, 130, 10524-10526.

26 E. C. Spencer, R. J. Angel, N. L. Ross, B. E. Hanson and J. A. K. Howard, J. Am. Chem. Soc., 2009, 131, 4022-4026.

27 A. J. Graham, D. R. Allan, A. Muszkiewicz, C. A. Morrison and S. A. Moggach, Angew. Chem., Int. Ed., 2011, 50, 11138-11141.

28 P. Serra-Crespo, E. Stavitski, F. Kapteijn and J. Gascon, RSC Adv., 2012, 2, 5051-5053.

29 K. J. Gagnon, C. M. Beavers and A. Clearfield, J. Am. Chem. Soc., 2013, 135, 1252-1255.

30 E. C. Spencer, M. Kiran, W. Li, U. Ramamurty, N. L. Ross and A. K. Cheetham, Angew. Chem., Int. Ed., 2014, 53, 5583-5586.
31 H. Wang, Z. G. Qu, W. Zhang, Q. N. Yu and Y. L. He, Int. J. Heat Mass Transfer, 2016, 92, 859-863.

32 H. K. Mao, J. Xu and P. M. Bell, J. Geophys. Res., [Solid Earth Planets], 1986, 91, 4673-4676.

33 A. I. Skoulidas, J. Am. Chem. Soc., 2004, 126, 1356-1357.

34 C. Prestipino, L. Regli, J. G. Vitillo, F. Bonino, A. Damin, C. Lamberti, A. Zecchina, P. L. Solari, K. O. Kongshaug and S. Bordiga, Chem. Mater., 2006, 18, 1337-1346.

35 S. Maiti, A. Pramanik, U. Manju and S. Mahanty, Microporous Mesoporous Mater., 2016, 226, 353-359.

36 J. Ethiraj, F. Bonino, C. Lamberti and S. Bordiga, Microporous Mesoporous Mater., 2015, 207, 90-94.

37 Y.-K. Seo, G. Hundal, I. T. Jang, Y. K. Hwang, C.-H. Jun and J.-S. Chang, Microporous Mesoporous Mater., 2009, 119, 331337. 